\title{
Autonomous lighting agents in global illumination
}

\author{
Adrien Herubel * \\ Institut Gaspard Monge
}

\author{
Venceslas Biri ${ }^{\dagger}$ \\ Institut Gaspard Monge
}

Farchad Bidgolirad
Duran Duboi
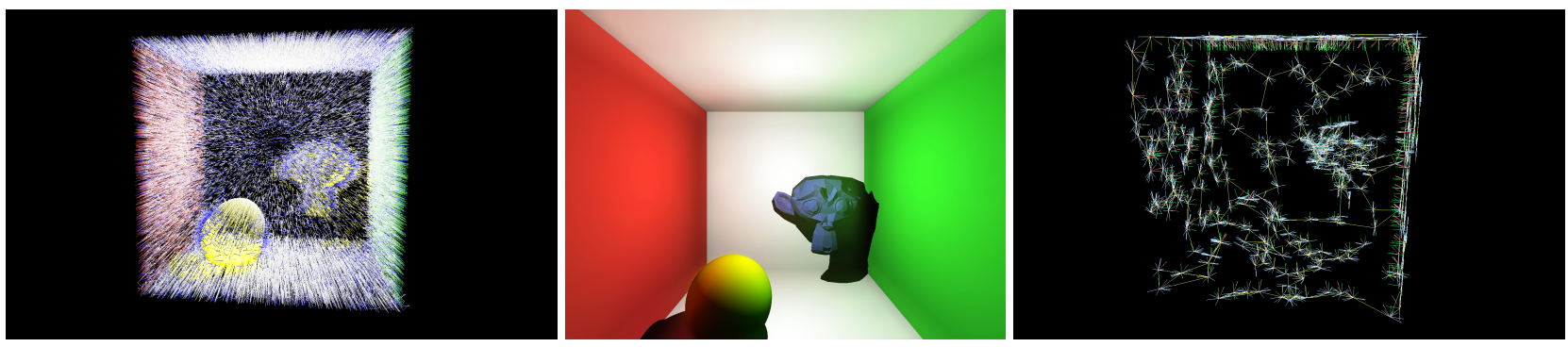

Figure 1: Traditional photon map, Rendered picture and Autonomous Lighting Agents networks

Keywords: global illumination, artificial intelligence, photon mapping

\section{Introduction}

In computer graphics, physically-based global illumination algorithms such as photon-mapping [2001] have a linear progression between complexity and quality. To a given quality, rendering time scales linearly with computer performances. With Moore's law call in question and increasing demand in quality, those algorithms need more and more optimisations.

Existing optimisations such as irradiance caching [1988] or shadow photons [1995] are themselves very linear in performance gain. They usually consists in adding knowledge about the scene and using it to interpolate previously computed values. Data is gathered in large volumes although due to heavy redundancy we observe a low density of useful data.

We propose a new optimisation method for the photon-mapping global illumination algorithm. We use structures borrowed from Artificial Intelligence such as autonomous agents to reduce computing time of the photon casting phase and rendering phase of the algorithm. Our structure called Autonomous Lighting Agents starts by using an agent-based scene discovery algorithm and is later used to make decisions during rendering inducing less photons being casted and shorter rendering times.

\section{Autonomous lighting agents}

Discovering the scene using autonomous agents We saw that optimisation techniques in photon-mapping use additional data about the scene. The information carried about the model in these optimisations is either too redundant or too local. In shadowphotons, a photon-map using 1 million photons can easily generate a 100k shadow photons in which only a small fraction is useful. In irradiance caching, data only consists in local distances and geometry gradients. Thus, to optimize the algorithm we developed a less uniform, and more data-dense global structure. Our structure borrows the properties of the photon-map but its goal is to gather data instead of carrying light flux. Efficiently discovering the scene implies a non-uniform data density. Data density should match rendering needs. Therefore, we decided to implement our method using artificial intelligence techniques. We use the autonomous agent software paradigm to discover the scene. Determining areas of interest should be without human intervention in order to be usable.

Our autonomous lighting agents efficiently gather large amount of useful data using various sensors and store it using decision-making algorithms. Agents exist within multiple graph representations. Each graph depicts relations between agents such as neighbourhood or light paths. The resulting structure is a network of agents storing data non-uniformly with increased density in zones of interest.

The oracle model The agent network is a complex structure to use during the rendering pass. To reduce the complexity we continue to use an agent based model. The raytracer uses this model like an oracle when a decision has to be taken. Our model implements answers to questions following the "Should I?" pattern. The raytracer questions the oracle, then the question is transferred to the most qualified to answer agent. The agent may transfer the question through its various networks and/or answer itself, then the answer is given to the raytracer.

\section{Results and discussion}

Our first results show an important decrease of memory occupation and slightly shorter rendering times for equal image quality. We plan to achieve significant gain of speed particularly in indirect diffuse illumination.

\section{References}

Jensen, H., And Christensen, N. 1995. Efficiently Rendering Shadows using the Photon Map. Compugraphics'95, 285-291.

Jensen, H. W. 2001. Realistic Image Synthesis Using Photon Mapping. A K Peters.

Ward, G., Rubinstein, F., And Clear, R. 1988. A ray tracing solution for diffuse interreflection. Proceedings of the 15th annual conference on Computer graphics and interactive techniques, 85-92.

\footnotetext{
*e-mail: herubel@igm.univ-mlv.fr

†e-mail:biri@univ-mlv.fr

‡e-mail:fbidgolirad@quintaindustries.com
} 